AEI-2005-015

\title{
A two-loop test for the factorised S-matrix of planar $\mathcal{N}=4$
}

\author{
B. Eden \\ Max-Planck-Institut für Gravitationsphysik, Albert-Einstein-Institut, \\ Am Mühlenberg 1, D-14476 Golm, Germany
}

\begin{abstract}
We check the recently proposed higher loop Bethe-ansatz for the $\operatorname{sl}(2)$ sector of $\mathcal{N}=4$ at two loops by a direct perturbative calculation using $\mathcal{N}=2$ superfields in supersymmetric dimensional reduction. Our method can in principle address operators built from many elementary fields and of arbitrary twist. We work out in detail the spin three, twist three operator mixing problem at leading order in $N$ and find agreement with the prediction based on integrability.
\end{abstract}




\section{Introduction}

The original version of the $\mathrm{AdS}_{5}-\mathrm{CFT}_{4}$ correspondence is a weak-strong duality so that a direct comparison of string theory with gauge theory quantities is hardly possible in the perturbative regime. In a special limit of the geometry this difficulty may have been overcome by the BMN-proposal [1]. The exact leading $N$ orthogonalisation of the relevant set of operators has been connected to integrable spin chains, thus opening the possibility of analysing the large $N$ mixing in $\mathcal{N}=4$ through the Bethe-ansatz [2]. The spin chain Hamiltonian can be identified with the dilatation operator of the planar CFT [3] , a powerful tool that has successfully been used e.g. to compute one- and two-loop scaling dimensions [4] of the gauge theory equivalent of a class of string theory solitons with large angular momentum [5].

On the field theory side, dilatation operator and Bethe-ansatz are known for the full theory at one-loop [6], while the dilatation operator has been worked out up to three loops for an $s u(2 \mid 3)$ subsector [7, and if the subalgebra is further restricted to $s u(2)$ also to four loops [8] and five loops [9]. For the $s u(2)$ sector a Bethe-ansatz up to three loops was formulated in [10] and there is even an all-loops conjecture based on a new long-range spin chain [9].

A step towards the quantisation of $\mathrm{AdS}_{5} \times \mathrm{S}_{5}$ string theory has been made in [1] by taking into account the lowest term in a perturbation series in the curvature around the $p p$-wave background underlying the BMN construction. Data from here as well as from the soliton calculations has been incorporated into a Bethe-ansatz on the string side [12]. The ansatz deviates from the gauge theory conjecture starting at three loop order in the gauge coupling, where there are extra scattering terms, although these are expressed in terms of quantities known from the gauge theory [9. The three-loop disagreement of the Bethe-ansaetze accurately reproduces the gauge theory/string theory mismatches found up to now [10, 11]. Despite of this, the integrable structures on the two sides of the conjecture are very similar: one may for example match the higher charges [13] at one and two loops. Furthermore, the spectrum of the classical string in the $s u(2)$ sector was derived from a set of integral Bethe-equations in 14, the equivalent of the thermodynamic limit of the discrete Bethe equations in the gauge theory picture. The idea behind the string Bethe-ansatz was in fact to look for an appropriate discretisation of the continuum equations. The ansatz has the feature of "factorised scattering", i.e. that the Bethe equations involve a product of two-site interactions termed $S$-matrices.

The article [16] shifts the attention entirely to the $S$-matrix. It is observed that the energy eigenvalues found in [1] for an $s u(1 \mid 1)$ part of the $s u(2 \mid 3)$ sector and in [15] for the disjoint $\operatorname{sl}(2)$ sector are compatible with factorised scattering. A relation between the string theory $S$-matrices in the three sectors is transferred to gauge theory, yielding a twoand three-loop Bethe-ansatz in the $s l(2)$ sector. The associated spectrum is checked for the twist two operators, whose anomalous dimensions up to three loops were extracted in 17. from an outstanding QCD calculation [18].

The purpose of the present paper is to test the two-loop anomalous dimensions (i.e. order $g^{4}$ ) given in [16] for the twist three, spin three operators in the $s l(2)$ sector against a direct calculation of two-point functions based on $\mathcal{N}=2$ superfields [19] and regularisation by supersymmetric dimensional reduction (SSDR) [20. ${ }^{1}$ The result strongly vindicates the

\footnotetext{
${ }^{1}$ Superspace calculations package ordinary Feynman graphs into supergraphs. In many situations this
} 
$S$-matrix conjecture of [16, since at least in the dilatation operator picture there is the issue of "wrapping", i.e. how the general interaction is modified when there are only few sites in the spin chain. We emphasize that our method is applicable beyond twist two; barring for computing limitations we can handle operators built from many elementary fields and carrying higher spins.

We encounter Feynman graphs with up to four loop integrations in momentum space. ${ }^{2}$ The only four-loop diagram is of a type that we call "BPS-like" since the same supergraphs without extra partial derivatives on the outer legs occur in the two-point functions of $1 / 2$ BPS operators. For the two- and three-loop diagrams of this type we verify finiteness with and without extra partial derivatives using the cutting edge Mincer algorithm [21]. By analogy, we assume that also the four-loop BPS-like supergraph does not contribute. On the other hand, all the singular integrals have maximally three loop integrations in momentum space so that they can be evaluated exactly.

Note that the Mincer programme itself calculates massless two-point integrals with two or three loop integrations and arbitrary scalar numerators. We reconstruct integrals with open indices from their projections with the total ingoing momentum.

Future work aims at the construction of the planar two-loop dilatation operator for the $\operatorname{sl}(2)$ sector, which would allow one to further analyse the recently discovered one-loop gauge/string theory discrepancies in the $s l(2)$ sector [22].

\section{The mixing problem}

We consider the leading $N$ mixing of the operators

$$
\left\{s_{1}, s_{2}, s_{3}\right\}=\operatorname{Tr}\left(\left(D_{z}^{s_{1}} Z\right)\left(D_{z}^{s_{2}} Z\right)\left(D_{z}^{s_{3}} Z\right)\right) .
$$

Here $Z$ is a complex scalar field of the $\mathcal{N}=4 \mathrm{SYM}$ theory with $S U(N)$ gauge group and $D_{\mu}=\partial_{\mu}+i g A_{\mu}$ is the Yang-Mills covariantised space-time derivative. The operators carry spin $s=s_{1}+s_{2}+s_{3}$, i.e. the Lorentz indices are symmetrised and traceless, which can be made automatic by projecting all indices onto $z=x_{1}+i x_{2}$ as indicated in (1).

The theory is regularised by SSDR (supersymmetric dimensional reduction) [20] in $x$ space. This scheme is related to dimensional regularisation, where the scalar propagator is

$$
\langle Z(1) \bar{Z}(2)\rangle=\frac{c_{0}}{x_{12}^{2}}\left(\mu x_{12}^{2}\right)^{\epsilon}, \quad c_{0}=-\frac{1}{4 \pi^{2}}, \quad \square_{1}\langle Z(1) \bar{Z}(2)\rangle=\delta\left(x_{12}\right) .
$$

(The normalisation change involving non-integer powers of $\pi$ and $\zeta(2)$ is hidden in the mass scale. We will not usually write $\mu$, since it can easily be reinstated.) On dimensional grounds, Feynman-graphs must come with the fractional powers

$$
g^{0}:\left(x_{12}^{2}\right)^{3 \epsilon}, \quad g^{2}:\left(x_{12}^{2}\right)^{4 \epsilon}, \quad g^{4}:\left(x_{12}^{2}\right)^{5 \epsilon},
$$

whose $\epsilon$-expansion yields logarithms. The conformal invariance of $\mathcal{N}=4 \mathrm{SYM}$ implies that these logs exponentiate to scaling dimensions associated with composite operators.

leads to a significant decrease of the number of diagrams. We prefer the $\mathcal{N}=2$ over the $\mathcal{N}=1$ formulation because the extra supersymmetry helps to suppress even more graphs.

${ }^{2}$ In the literature the $O\left(g^{2}\right), O\left(g^{4}\right)$ parts of the scaling dimension are usually called one-loop and two-loop anomalous dimension. Nevertheless, when the first two anomalous dimensions are computed from two-point functions the momentum space Feynman integrals have two loops at order $g^{2}$ and up to four at $g^{4}$. 
Such conformal properties become visible only in the renormalised theory, i.e. after introduction of $\mathcal{Z}$-factors and in the limit $\epsilon \rightarrow 0$. The set of operators has to be orthogonalised, upon which the two-point function of a correctly renormalised primary of spin $s$ has the form

$$
\left\langle P^{s}(1) \bar{P}^{s}(2)\right\rangle=\frac{c\left(g^{2}\right) J_{\mu_{1} \nu_{1}}\left(x_{12}\right) \ldots J_{\mu_{s} \nu_{s}}\left(x_{12}\right)}{\left(x_{12}^{2}\right)^{\Delta\left(g^{2}\right)}}
$$

where the sets of indices $\left\{\mu_{1} \ldots \mu_{s}\right\}$ and $\left\{\nu_{1} \ldots \nu_{s}\right\}$ are both individually symmetrised and made traceless [23]. In the last equation we have used the inversion tensor

$$
J_{\mu \nu}(x)=\eta_{\mu \nu}-2 \frac{x_{\mu} x_{\nu}}{x^{2}}
$$

to organise the terms in a simple manner. The scaling dimension $\Delta=\Delta_{0}+g^{2} \gamma_{1}+g^{4} \gamma_{2}+\ldots$ splits into a classical part $\Delta_{0}$ and the "anomalous dimension" depending on $g^{2}$. It can clearly be read off from any term in (4). We find it convenient to drop all traces because this reduces the set of graphs, see below. If desired, the full functional form of any two-point function can be reconstructed: for primary operators we may use (4) while two-point functions of conformal descendants are obtained from it by differentiation.

We are interested in the anomalous dimension up to order $g^{4}$, which requires the leading and the next-to-leading term in the $\epsilon$-expansion, i.e.

$$
g^{0}: O(1), O(\epsilon), \quad g^{2}: O\left(\frac{1}{\epsilon}\right), O(1), \quad g^{4}: O\left(\frac{1}{\epsilon^{2}}\right), O\left(\frac{1}{\epsilon}\right) .
$$

In particular, at $g^{4}$ we do not need to keep track of the finite part of the integrals. Correspondingly, we will not eliminate finite $O\left(g^{4}\right)$ cross terms between the operators. Terms of order $\epsilon$ and higher yield contact terms when the regulator is taken off [24]. Although we are generally not interested in the contact part we cannot discard the $O(\epsilon)$ terms in the tree-level mixing, because the singular renormalisation factors relate them to the subleading terms at $g^{2}$ and $g^{4}$. The renormalisation/orthogonalisation proceeds along these lines: first, we need to choose a basis in which the $O(1)$ tree-level mixing and the simple logarithm terms at $g^{2}$ are diagonalised. In such a basis each operator picks up a $\mathcal{Z}$-factor of the standard form 25 ]

$$
\mathcal{Z}=1+g^{2} \frac{\gamma_{1}}{2 \epsilon}+g^{4}\left(\frac{\gamma_{1}^{2}}{8 \epsilon^{2}}+\frac{\gamma_{2}}{4 \epsilon}\right)+O\left(g^{6}\right)
$$

and $\gamma_{1}, \gamma_{2}$ can already be calculated. Second, in order to fully orthogonalise we may have to subtract the other operators with mixing coefficients of the type

$$
\mathcal{B}=g^{2} b_{10}+g^{4}\left(\frac{b_{21}}{\epsilon}+b_{20}\right)+O\left(g^{6}\right)
$$

(In this letter $b_{20}$ remains undetermined because we truncate the $\epsilon$-expansion.) This form of the $\mathcal{Z}$-factors may be called "minimal subtraction" since we strictly eliminate only singularities and finite cross terms.

At spin $\mathbf{0}$ we have the operator

$$
O=\{0,0,0\}
$$


At spin 1 we find its descendant

$$
D_{z} O=\{1,0,0\}
$$

At spin 2 there are

$$
\begin{aligned}
D_{z}^{2} O & =\{2,0,0\}+2\{1,1,0\}, \\
K_{6} & =\{2,0,0\}-(2+\epsilon)\{1,1,0\} .
\end{aligned}
$$

The operator $O$ is a $1 / 2$ BPS state. It is gratifying to see that its $g^{2}, g^{4}$ two-point functions do indeed not contain singular or finite pieces. For $x_{1} \neq x_{2}, \epsilon \rightarrow 0$ the loop contributions to $\langle O \bar{O}\rangle$ converge to zero and thus our calculation confirms protectedness. Let the descendants $D_{z}^{s} O, s=1,2,3$ be defined by naive distribution of each derivative over the three sites in the operator. All three descendants are exact null-vectors of the $g^{2}$ and $g^{4}$ mixing.

At spin 2 there is a second operator which we must orthogonalise w.r.t. $D_{z}^{2} O$. The definition (11) for $K_{6}$ is not quite in the spirit of minimal subtraction because we have used a mixing coefficient involving a positive power of $\epsilon$. It has the advantage that the bare $K_{6}$ is tree-orthogonal to $D_{z}^{2} O$ at leading and next-to-leading order in $\epsilon$, whereas this is guaranteed at $g^{2}, g^{4}$. Thus with the choice (111) the renormalised operator $K_{6 \text { ren }}=\mathcal{Z}_{K} K_{6}$ decouples from $D_{z}^{2} O$. Using a standard $\mathcal{Z}$-factor we find

$$
\left\langle K_{6 r e n} \bar{K}_{6 r e n}\right\rangle=-128 M^{3}\left(1+g^{2} M+\ldots\right) \frac{x_{12 z} x_{12 z} x_{12} x_{12} \bar{z}}{\left(x_{12}^{2}\right)^{\left(7+4 g^{2} M-6 g^{4} M^{2}+\ldots\right)}}-\text { traces }
$$

with the loop-counting factor $M=N /\left(4 \pi^{2}\right)$. The values

$$
\gamma_{1}=4 M, \quad \gamma_{2}=-6 M^{2}
$$

clearly identify the operator $K_{6}$ as belonging to the BMN-multiplet starting with the superprimary state $\operatorname{Tr}\left(Z \Phi_{I} \bar{\Phi}^{I}\right)+\operatorname{Tr}\left(Z \bar{\Phi}^{I} \Phi_{I}\right)$ analysed in [3, 25]. ${ }^{3}$

For minimal subtraction in the tightest sense we might choose $K_{6}^{\prime}=\{2,0,0\}-2\{1,1,0\}$. The divergence in $\mathcal{Z}_{K}$ then sees the $O(\epsilon)$ term in $\left\langle K_{6}^{\prime} D_{z}^{2} O\right\rangle$ and forces a renormalisation

$$
K_{6 r e n}=\mathcal{Z}_{K} K_{6}^{\prime}-\left(g^{2} \frac{M}{2}+g^{4}\left(\frac{M^{2}}{2 \epsilon}+b_{20}\right)+O\left(g^{6}\right)\right) D_{z}^{2} O
$$

where $\mathcal{Z}_{K}$ still has standard form. This is not multiplicative as $\mathcal{Z}_{K}=1+2 g^{2} M / \epsilon+\ldots$. To the order we work there is apparently freedom in the system, the stable feature being the anomalous dimension of $K_{6}$.

For the spin 3 mixing problem we return to our somewhat more elegant first scheme, namely to make the operators tree-orthogonal at leading and next-to-leading order. This is achieved by

$$
\begin{aligned}
D_{z}^{3} O & =\{3,0,0\}+3(\{2,1,0\}+\{1,2,0\})+2\{1,1,1\} \\
D_{z} K_{6} & =\{3,0,0\}-(1+\epsilon)(\{2,1,0\}+\{1,2,0\})-(2+\epsilon)\{1,1,1\}, \\
V_{1} & =\left(2-\frac{68 \epsilon}{35}\right)\{3,0,0\}-\left(9-\frac{96 \epsilon}{35}\right)(\{2,1,0\}+\{1,2,0\})+\left(24+\frac{164 \epsilon}{35}\right)\{1,1,1\}, \\
V_{2} & =\{2,1,0\}-\{1,2,0\} .
\end{aligned}
$$

\footnotetext{
${ }^{3}$ The convention used in 25] differs by a rescaling of the coupling constant.
} 
The operator $V_{2}$ is the only one that is odd under reversal of the three sites in the trace whereby it completely decouples at classical and quantum level. The first two operators $D_{z}^{3} O$ and $D_{z} K_{6}$ are defined by naive differentiation of their spin 2 ancestors. As already mentioned, $D_{z}^{3} O$ is a null-vector of the loop-level mixing; in conclusion this operator does not mix either.

One may wonder what correlation functions $D_{z} K_{6}$ and $V_{1}$ have w.r.t. the conformal primary $K_{6}$ itself. By direct calculation

$$
\left\langle K_{6 \text { ren }} D_{\bar{z}} \bar{K}_{6 r e n}\right\rangle=-1792 M^{3}\left(1+\frac{11}{7} g^{2} M+\ldots\right) \frac{x_{12 z} x_{12 z} x_{12 \bar{z}} x_{12 \bar{z}} x_{12 \bar{z}}}{\left(x_{12}^{2}\right)^{\left(8+4 g^{2} M-6 g^{4} M^{2}+\ldots\right)}}-\text { traces }
$$

as expected by differentiating formula (12) ${ }^{4}$ More interesting is the question as to how $V_{1}$ can be made orthogonal to $K_{6}$. We start with the definition

$$
V_{1 \text { ren }}=\mathcal{Z}_{V} V_{1}-\mathcal{B} D_{z} K_{6}
$$

and impose

$$
\left\langle V_{1 \text { ren }} \bar{K}_{6 r e n}\right\rangle=0 .
$$

Here $K_{6 \text { ren }}$ must be as before, while we take $\mathcal{Z}_{V}, B$ in standard form (7), (8) but with unknown coefficients. The simultaneous expansion of (18) in $\epsilon$ and $g^{2}$ yields three conditions from the vanishing of the constant term at $g^{2}$ and the pole and the simple logarithm at $g^{4}$. The result is

$$
\mathcal{Z}_{V}=1+g^{2} \frac{15 M}{4 \epsilon}+O\left(g^{4}\right), \quad \mathcal{B}=g^{2} \frac{15 M}{7}+g^{4}\left(\frac{345 M^{2}}{56 \epsilon}+b_{20}\right)+O\left(g^{6}\right) .
$$

In the spin $3 /$ spin 3 mixing $V_{1 \text { ren }}$ stays orthogonal to $D_{z} K_{6}$. The requirement of finiteness of $\left\langle V_{1 \text { ren }} \bar{V}_{1 \text { ren }}\right\rangle$ allows one to fix the $g^{4}$ part of $\mathcal{Z}_{V}$ which is in fact of the form (7).

Last, $V_{2}$ may be renormalised multiplicatively as it does not mix. Our main result is that $V_{1}, V_{2}$ have identical anomalous dimensions

$$
\gamma_{1}=\frac{15}{2} M, \quad \gamma_{2}=-\frac{225}{16} M^{2},
$$

i.e. the values predicted in [16].

\section{The graph calculation with $\mathcal{N}=2$ superfields}

\subsection{A shopping spree in $\mathcal{N}=2$ land}

The $\operatorname{sl}(2)$ sector contains the operators defined in (11), though in general with $k$ insertions of elementary fields that may all carry covariant derivatives. Independently of the spin, the operators are in the same $[0, k, 0]$ representations of $S U(4)$ as the $1 / 2$ BPS states without derivatives. The definition (10) is a highest weight projection in an $\mathcal{N}=1$ formulation, while

\footnotetext{
${ }^{4}$ Likewise, the direct evaluation of $\left\langle D_{z} K_{6 \text { ren }} D_{\bar{z}} \bar{K}_{6 \text { ren }}\right\rangle$ yields the expected $x$-dependence with the correct normalisation $28672 M^{3}\left(1+29 / 14 g^{2} M+\ldots\right)$. The point may be worth mentioning as the projection method in the graph calculation is relatively involved.
} 
in $\mathcal{N}=2$ we may realise representatives as chains of $k$ copies of the hypermultiplet $q^{+}$. One returns to the physical scalars of the $\mathcal{N}=4$ multiplet by putting $\theta=0$. The $\mathcal{N}=2$ two-point functions of composites of matter fields involve relatively few supergraphs. The advantage is quite clear: at $O\left(g^{2}\right)$ we have only one contributing diagram and at $g^{4}$ there are no more than fifteen singular graphs, which can be evaluated by standard means since they lead to two-point integrals up to and including three-loop level in momentum space.

Quantum calculations for the BPS operators themselves give zero, but divergences do arise when the hypermultiplets (or $Z$ 's) carry Yang-Mills covariant derivatives. We find that the partial derivative in $\mathcal{D}_{\mu}=\partial_{\mu}+i g A_{\mu}$ does not introduce singularities but that the connection part gives rise to a set of singular diagrams. This seems logical since the hypermultiplet itself and its partial derivatives are short objects from an $\mathcal{N}=2$ perspective while the connection is not.

We will not review the $\mathcal{N}=2$ formalism in this letter; the interested reader is referred to [19. Our conventions are taken from the second reference therein. The $\mathcal{N}=2 x$-space supergraph technique was developed in the series of papers [26]. The $\mathcal{N}=4$ action is

$$
\begin{aligned}
S_{N=4} & =S_{q}+S_{V}+\text { ghost }+ \text { gauge-fixing } \\
S_{q} & =-\operatorname{Tr}\left(\int d u d \zeta^{-4}\left(\tilde{q}^{+} D^{++} q^{+}+g i \tilde{q}^{+}\left[V^{++}, q^{+}\right]\right)\right) \\
S_{V} & =\frac{1}{2 g^{2}} \sum_{n=2}^{\infty} \frac{(-i g)^{n}}{n} \operatorname{Tr}\left(\int d^{12} z d u_{1} \ldots d u_{n} \frac{V^{++}\left(z, u_{1}\right) \ldots V^{++}\left(z, u_{n}\right)}{(12)(23) \ldots(n 1)}\right)
\end{aligned}
$$

with $\zeta=\left\{x_{A}, \theta^{+}, \bar{\theta}^{+}\right\}$and $z=\{x, \theta, \bar{\theta}\}$. The "harmonics" $u \in S U(2)$ are auxiliary bosonic variables. We use the shorthand $(23)=u_{2}^{+i} u_{3}^{+}$, where 2,3 label distinct points and $i \in$ $\{1,2\}$, while the row index is split into $\{+,-\}$ according to the charge under the $U(1)$ subgroup. The $u$-variable is used as a projector: $\theta^{ \pm}=u_{i}^{ \pm} \theta^{i}$ and similarly for $\bar{\theta}$ and the spinorial derivatives $D, \bar{D}$. The "analytic $x$ "

$$
x_{A}^{\mu}=x^{\mu}-i\left(\theta^{+} \sigma^{\mu} \bar{\theta}^{-}+\theta^{-} \sigma^{\mu} \bar{\theta}^{+}\right)
$$

is annihilated by $D^{+}, \bar{D}^{+}$. In the YM action the $u$-variables distinguish the various $V$ fields. The $S U(2)$ structure of the three-vertex is in fact completely antisymmetric so that the simple product from (23) becomes a commutator.

The two unconstrained quantum fields of the formalism are the hypermultiplet $q^{+}\left(x_{A}, \theta^{+}\right.$, $\left.\bar{\theta}^{+}, u\right)$ and the YM prepotential $V^{++}\left(x_{A}, \theta^{+}, \bar{\theta}^{+}, u\right)$. We have the propagators

$$
\begin{aligned}
\Pi_{12} & =\left\langle q^{+}(1) \tilde{q}^{+}(2)\right\rangle=e^{\Delta_{12}} \frac{1}{-4 \pi^{2} x_{A 1 A 2}^{2}}, \\
\Delta_{12} & =\frac{2 i}{(12)}\left[\left(1^{-} 2\right) \theta_{1}^{+} \partial_{1} \bar{\theta}_{1}^{+}+\left(2^{-} 1\right) \theta_{2}^{+} \partial_{1} \bar{\theta}_{2}^{+}+\theta_{1}^{+} \partial_{1} \bar{\theta}_{2}^{+}+\theta_{2}^{+} \partial_{1} \bar{\theta}_{1}^{+}\right],
\end{aligned}
$$

and in Feynman gauge

$$
\left\langle V^{++}(1) V^{++}(2)\right\rangle=\delta\left(u_{1}, u_{2}\right) \frac{\left(\theta_{1}^{+}-\theta_{2}^{1+}\right)^{2}\left(\bar{\theta}_{1}^{+}-\bar{\theta}_{2}^{1+}\right)^{2}}{2 \pi^{2} x_{12}^{2}} .
$$

With the notation $\theta_{2}^{1+}$ we mean $\theta$ at point 2 , projected by $u^{+}$from point 1 . (The expression for the propagator remains reasonable due to the presence of the $S U(2)$ delta-function.) 
Note that there is no higher matter/YM interaction than the cubic term in $S_{q}$, while there exist YM self-interactions of any order. Our set of graphs involves only the cubic YM vertex, though. From the interaction Lagrangian we retain

$$
\begin{aligned}
& -g i \int d u d \zeta^{-4} \operatorname{Tr}\left(\tilde{q}^{+}\left[V^{++}, q^{+}\right]\right) \\
& \frac{g i}{12} \int d^{12} z d u_{1} d u_{2} d u_{3} \frac{\operatorname{Tr}\left(V^{++}\left(z, u_{1}\right)\left[V^{++}\left(z, u_{2}\right), V^{++}\left(z, u_{3}\right)\right]\right)}{(12)(23)(31)}
\end{aligned}
$$

The SSDR scheme means to leave the spinor and $S U(2)$ algebra untouched but to modify the $1 / x^{2}$ part of the propagators as in (2).

Finally, we need to discuss the connection in $\mathcal{D}_{\mu}=\partial_{\mu}+g i A_{\mu}$. In the $\lambda$-frame [19] we have

$$
\mathcal{D}_{\alpha}^{+}=D_{\alpha}^{+}, \quad \overline{\mathcal{D}}_{\alpha}^{+}=\bar{D}_{\alpha}^{+} .
$$

(Hence the + projected spinor derivatives are YM covariant.) The - projected spinor derivatives inherit a connection from the $S U(2)$ derivative $\mathcal{D}^{--}$:

$$
\begin{aligned}
\mathcal{D}^{--} & =D^{--}+g i V^{--} \\
\mathcal{D}_{\alpha}^{-} & =\left[\mathcal{D}^{--}, D_{\alpha}^{+}\right]=D_{\alpha}^{-}-g i\left(D_{\alpha}^{+} V^{--}\right) \\
\overline{\mathcal{D}}_{\dot{\alpha}}^{-} & =\left[\mathcal{D}^{--}, \bar{D}_{\dot{\alpha}}^{+}\right]=\bar{D}_{\dot{\alpha}}^{-}-g i\left(\bar{D}_{\dot{\alpha}}^{+} V^{--}\right)
\end{aligned}
$$

The super-algebra does not change under covariantisation:

$$
\left\{\mathcal{D}_{\alpha}^{+}, \overline{\mathcal{D}}_{\dot{\alpha}}^{-}\right\}=-2 i \mathcal{D}_{\alpha \dot{\alpha}}
$$

from where we may read off

$$
\mathcal{D}_{\mu}=\partial_{\mu}+\frac{g}{4}\left(D^{+} \sigma_{\mu} \bar{D}^{+} V^{--}\right)
$$

The field $V^{--}$has a series expansion in terms of $V^{++}$quite like the YM action:

$$
\begin{aligned}
V^{--}\left(z, u_{1}\right) & =\int d u_{2} \frac{V^{++}\left(z, u_{2}\right)}{(12)^{2}}+ \\
& +\frac{g i}{2} \int d u_{2} d u_{3} \frac{\left[V^{++}\left(z, u_{2}\right), V^{++}\left(z, u_{3}\right)\right]}{(12)(23)(31)}+\ldots
\end{aligned}
$$

We draw the reader's attention to the non-linearity of the connection (34) in $V^{++}$. For our purposes only the first two terms of the expansion are needed. Note that the linear term has the following two-point function with $V^{++}$:

$$
\left\langle\frac{1}{4}\left(D^{+} \sigma_{\mu} \bar{D}^{+} V^{--}\right)(1) V^{++}(2)\right\rangle_{\theta_{1}, \bar{\theta}_{1}=0}=-e^{\frac{2 i}{(12)}\left(2^{-} 1\right) \theta_{2}^{+} \partial_{1} \bar{\theta}_{2}^{+}} \frac{\theta_{2}^{+} \sigma_{\mu} \bar{\theta}_{2}^{+}}{2 \pi^{2}\left(x_{1}-x_{A 2}\right)^{2}}
$$

When evaluating Feynman graphs with connection ends it is a very useful fact that the exponential shift in this formula coincides with one of the terms in the shift acting on the matter propagator. This exponential is usually reproduced also in graphs involving the 
quadratic part in the connection because cross terms from the denominators of the attached propagators are eliminated when putting the outer $\theta$ 's to zero.

Let us comment on how to evaluate supergraphs. A YM-line between two matter/YM vertices is relatively easy to deal with: owing to the presence of the $u$ delta-function and the nilpotent numerator we may change the denominator of the $V$-propagator from $x_{1}-x_{2}$ to $x_{A 1}-x_{A 2}$. One $S U(2)$ integration can trivially be done using the delta-function. We may now use the numerator of the propagator as a delta-function for the + projection of the two $\theta$ 's, the minus projection is absent. Thus we replace, say the spinors in the exponential shifts $\Delta$ at the second vertex by those from the first and integrate away the delta-function. The second $\theta$ and $u$ integration is left over for the moment.

Graphs involving the cubic YM vertex or a connection end are trickier in that a given $\theta$ may occur in contractions w.r.t. several different $u$ 's. If the YM lines end on matter lines it can be an advantage to shift to a chiral basis at the complicated end, otherwise it is hard to give general rules. In order to integrate out the $\theta$ at the cubic vertex it is always advisable to choose that projector which occurs most often and to use the "cyclic identity" 5 to write all occurrences of the $\theta$ in question in terms of the,+- projections w.r.t. this one harmonic.

Eventually one has to expand the exponentials and the numerators in the remaining $\theta$ 's, leading to a linear combination of differential operators under the $x$-integrations with harmonic (i.e. $S U(2)$ ) integrals as coefficients. These $u$-integrations can always be done in an algebraic fashion: if there is no denominator one can straightforwardly express them in terms of $S U(2)$ epsilon tensors, else one pulls a $D^{++}$off the numerator and partially integrates it onto the denominator of the integral thus producing delta-functions [19]. For the more complex $g^{4}$ supergraphs we admittedly had to resort to a Mathematica programme to do parts of the Grassmann algebra and the $S U(2)$ integrations.

In the pictures below we have split the lines emanating from the outer points. We do so purely for convenience of drawing - all lines coming in from the left start at $z_{1}=$ $\left\{x_{1}, \theta_{1}, u_{1}\right\}$ and all lines ending on the right come together at the point with coordinates $z_{2}=\left\{x_{2}, \theta_{2}, u_{2}\right\}$. "Spectator lines", i.e. free matter propagators have been omitted. There are, of course, as many as needed to connect all matter fields in the composite operators at points $z_{1}, z_{2}$. We did not indicate the outer partial derivatives either since they do not interfere with the Grassmann- and $S U(2)$-integrations.

Note that any two-point graph related to a $[0, k, 0]$ operator will come with a factor $(12)^{k}$, which we simply drop. It is equal in the tree-level and the $g^{2}$ and $g^{4}$ parts and universal to all operators built from $k$ fields; therefore it does not influence the renormalisation at all. At order $g^{4}$ individual graphs also produce "harmonic-nonanalytic" terms coming with $(12)^{k+1}\left(1^{-} 2^{-}\right)$, which must eventually cancel. A good sign is that all such terms contain free propagators times the finite integral $f$ from graph $B_{0}$ below. We did not explicitly check the vanishing of their sum because they are irrelevant to the second anomalous dimension.

\footnotetext{
${ }^{5}$ For three harmonics: $(12) u_{3}+(23) u_{1}+(31) u_{2}=0$, similarly with one or several - projections.
} 


\subsection{Graphs}

At order $g^{2}$ there are only two graphs, for which we find the $x$-space expressions

$$
\begin{aligned}
& B_{0}=-(12)^{2} \square_{1} f(1,2 ; 1,2), \\
& G_{0}=-(12) i \partial_{2}^{\mu} g(1,1,2) .
\end{aligned}
$$

Here we have omitted the normalisation constants of the propagators, i.e. $c_{0}=-1 /\left(4 \pi^{2}\right)$ from the hypermultiplet propagator and $-2 c_{0}$ from the normalisation of the $V$-line. The integrals $f, g$ are as the pictures suggest - there is no extra numerator and all lines are to be replaced by $1 / x_{i j}^{2}$, hence

$$
f(1,2 ; 1,2)=\int \frac{d^{4} x_{3} d^{4} x_{4}}{x_{13}^{2} x_{14}^{2} x_{34}^{2} x_{23}^{2} x_{24}^{2}}, \quad g(1,1,2)=\int \frac{d^{4} x_{3}}{\left(x_{13}^{2}\right)^{2} x_{23}^{2}} .
$$

The topology of a graph cannot change due to the spinor and $S U(2)$ algebra other than by going into a "derived topology", i.e. one in which some lines are shrunk to points by box operators.
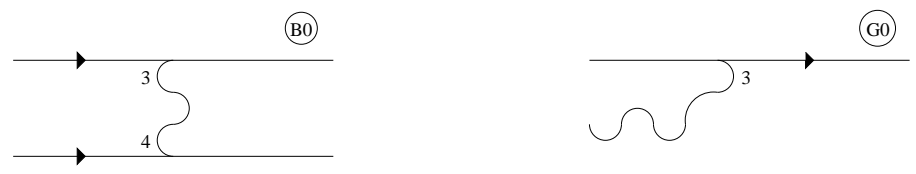

Figure 1. Graphs at order $g^{2}$

Let us focus on diagram $B_{0}$. The double-box integral $f$ is finite and by dimensionality we have $f(1,2 ; 1,2) \propto 1 / x_{12}^{2}$, so that the outer box operator produces a delta-function. In the SSDR scheme this is seen as an $O(\epsilon)$ term, see Section 2. This would be the only contribution in a two-point function of products of hypermultiplets without any derivatives. Indeed, such operators are $1 / 2$ BPS and their two-point functions should not receive any quantum corrections except for contact terms.

What happens when the diagram has partial derivatives on the outer legs? The Mincer system can calculate $f$ in momentum space, and it can also handle arbitrary scalar numerators. We use the programme according to the following strategy:

- All partial derivatives are translated into momentum space as momenta with open indices. We make the sets of indices symmetric and traceless separately at the left and at the right end of the graph, so that they define spin $s_{l}$ and spin $s_{r}$ "harmonic tensors", respectively.

- There are $\operatorname{Min}\left(s_{l}, s_{r}\right)+1$ independent ways of projecting this with the total ingoing momentum $q$, because we may choose to contract 0 through $\operatorname{Min}\left(s_{l}, s_{r}\right)$ indices with the metric $\eta$. All remaining open indices are contracted on $q$.

- For any given distribution of outer partial derivatives/momenta we calculate all such projections by Mincer.

- The integral with open indices (symmetrised and traceless at each end) contains exactly as many independent Lorentz structures as there are projections, the criterion 
being once again how many $\eta$ symbols with one index from the left set and one from the right set are involved. It is a simple exercise to set up and invert the set of linear equations which allows to reconstruct the coefficient of each basis element from the projections. The coefficients are generally $\epsilon$-dependent; we keep $O(1), O(\epsilon)$.

- We Fourier-transform back to $x$-space.

In this context, too, it is actually most convenient to discard all traces since under the FT

$$
(i q)^{\left(s_{l}+s_{r}\right)}\left(q^{2}\right)^{-2 \epsilon} \rightarrow \frac{2 \epsilon(1-3 \epsilon+\ldots)}{\pi^{2}}\left(-\partial_{1}\right)^{\left(s_{l}+s_{r}\right)} \frac{1}{\left(x_{12}^{2}\right)^{(2-3 \epsilon)}}
$$

and the term without $\eta$ is simply obtained by having all derivatives act on the denominator.

For $B_{0}$ we have scanned all spins up to three derivatives at one end and four at the other. Our findings are surprising: the term without $\eta$ remains a contact term for any harmonic tensors at the left and at the right end, while terms with $\eta$ may be finite. In conclusion, the "BPS-like" diagram $B_{0}$ drops out of the calculation of the first anomalous dimension.

The calculation of $G_{0}$ can be done in a variety of ways because the underlying integral $g(1,1,2)$ is elementary. Since our computer programmes were originally geared at the $[0,2,0]$ operators we have chosen to join one free line and compute the resulting two-loop diagram as a derived topology of the T2 graph [21, alternatively one may use T1. The results of Mincer have to be scaled down by a factor $(4 \pi)^{2 l}$ where $l$ is the loop order of the given integral. In configuration space graph $G_{0}$ has a simple pole. ${ }^{6}$

We remark that the diagrams have to be recomputed for every different distribution of derivatives. The combinatorics becomes more elaborate, too, since equal looking legs can be distinguished by the number of partial derivatives.

On the computing side, we have implemented the large $N$ combinatorics for the $[0,3,0]$ operators in a Mathematica programme, which uses a table of integrals to calculate the matrices of two point functions at tree-level and orders $g^{2}, g^{4}$. The calculation of each integral requires the construction of the necessary harmonic tensors and their projections; for convenience of programming we still do this under Mathematica. Finally, we link in Mincer under Form to evaluate the projected integrals.

Let us switch to order $g^{4}$. There are five "BPS-like" graphs, i.e. topologies that one also finds in two-point functions of $1 / 2$ BPS operators, though without extra partial derivatives on the outer legs. When analysing two-point functions of $1 / 2$ BPS operators one tends to find two linear combinations of graphs coming with independent colour structures [24]. Protectedness is tantamount to both of these linear combinations being contact terms. In the manifestly finite setup we use $(\mathcal{N}=4$ in terms of $\mathcal{N}=2$ fields $)$ the only divergences are associated to composite operators. Therefore, graphs related to BPS objects should even be individually finite.

The BPS-like supergraphs are drawn in Figure 2. In $B_{2}$ there is no vertex in the middle of the diagram, likewise in $B_{5}$ the YM line between points 5 and 6 does not touch the matter line in the middle of the diagram.

\footnotetext{
${ }^{6}$ The index of the connection "migrates" to the opposite end of the graph, see (39). When building up the harmonic tensors we must put this index into the tensor at the left end, of course.
} 

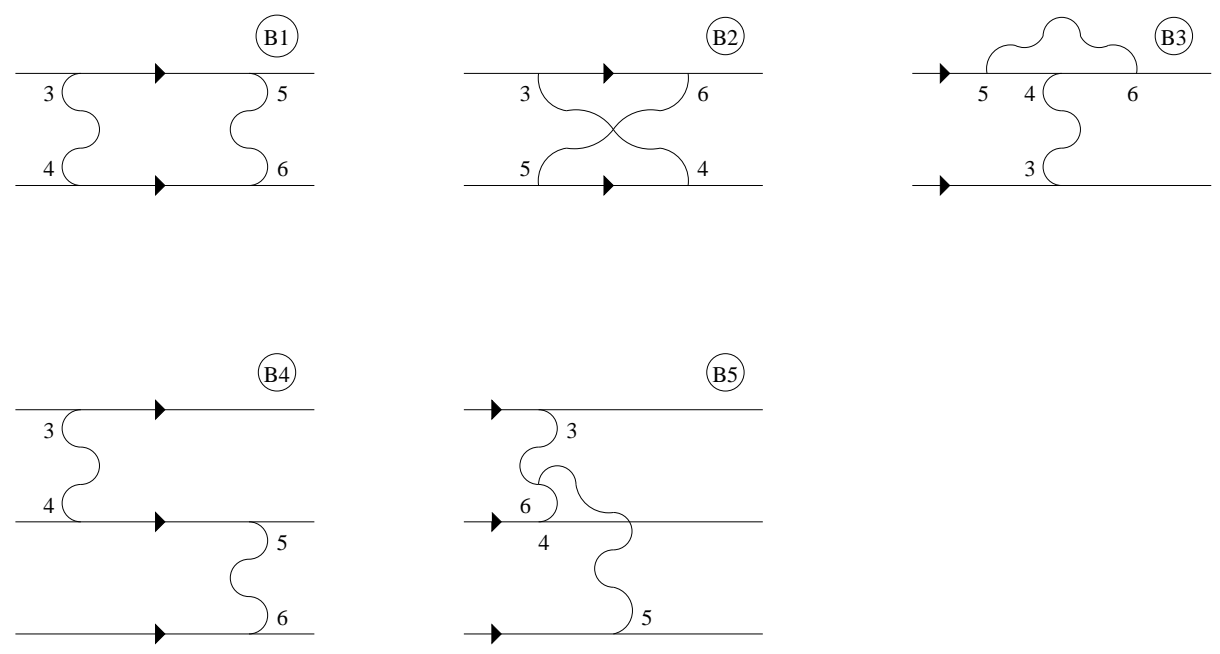

Figure 2. BPS-like graphs at order $g^{4}$.

We find

$$
\begin{aligned}
B_{1}= & (12)^{2} \square_{1} \square_{1} L A, \\
B_{2}= & (12)^{2}\left[-\square_{1}\left(\partial_{13}+\partial_{24}\right)^{2}+(12)\left(1^{-} 2^{-}\right)\left(\square_{15} \square_{24}+\square_{13} \square_{26}\right)\right] N P, \\
B_{3}= & (12)^{2}\left[-\square_{1} \square_{34}+(12)\left(1^{-} 2^{-}\right)\left(\square_{15} \square_{23}+\square_{13} \square_{26}\right)\right] B E, \\
B_{4}= & (12)^{3}\left[\left(\partial_{13}+\partial_{14}\right)^{2}\left(\partial_{25}+\partial_{26}\right)^{2}-\square_{1} \square_{45} / 2-\square_{16} \square_{23}+\right. \\
& \left.\quad+(12)\left(1^{-} 2^{-}\right) \square_{16} \square_{23}\right] P 4,
\end{aligned}
$$

where the normalisation constants of the propagators were again omitted. The $x$-space integrals $L A, N P, B E$ are the so-called "ladder", "non-planar" and "Benz" three-loop integrals as depicted in Figure 2. We illustrated by equation (40) how to read them off from the graphics; there is no additional numerator and the denominator is given by the appropriate product of $1 / x_{i j}^{2}$, no propagator is doubled. By $\partial_{i j}, \square_{i j}$ we mean derivatives acting at point $i$ on a line going from $i$ to $j$, whereas $\square_{1}$ means an outer box-operator acting on the whole expression.

All three topologies $L A, N P$ and $B E$ are finite without a numerator, but differentiation under the integral could potentially make them divergent. Using the Mincer system we have checked that the complete expressions $B_{1}, B_{2}, B_{3}$ do not diverge either. Like $B_{0}$ the graphs remain finite, too, when symmetrised, traceless products of derivatives are introduced acting from the left and from the right, respectively. A scan found no singularities up to spin 3 at one end and spin 4 at the other.

The integral in graph $B_{4}$ was baptised "P4" above as it is planar and has four loops. A quick evaluation of (45) in point-splitting suggests that the graph without extra derivatives is finite. Unfortunately, we have no way of calculating $B_{4}$ in dimensional regularisation, in particular in the presence of outer partial derivatives. By analogy with $B_{0} \ldots B_{3}$ we assume that $B_{4}$ is finite, too, so that we may dispose of it. Diagram $B_{5}$ should behave accordingly; at any rate, as a non-planar structure it is not relevant in the context of our leading $N$ analysis.

Note that the non-analytic parts in (43) ... (45) always reduce to $f(1,2 ; 1,2)$ whence they are finite by what was said about $B_{0}$. 
The singular graphs at order $g^{4}$ are listed in Figure 3. We have omitted the mirror graphs and some diagrams that must carry at least one power of $\eta$ on dimensional grounds, e.g. the graph with three connection lines joining at a cubic YM vertex. The product $B_{0} * G_{0}$ has no singular term without $\eta$ either, see above.

The graphs in the third row carry an index $\mu$ at point 1 on the left and an index $\nu$ at point 2 on the right. The dot in $G_{4}, G_{7}, G_{8}$ indicates the quadratic term in the connection from which two $V$-lines emanate, whereas $G_{13}, G_{14}, G_{15}$ have two different occurrences of the connection on the left with the indices $\mu, \nu$.
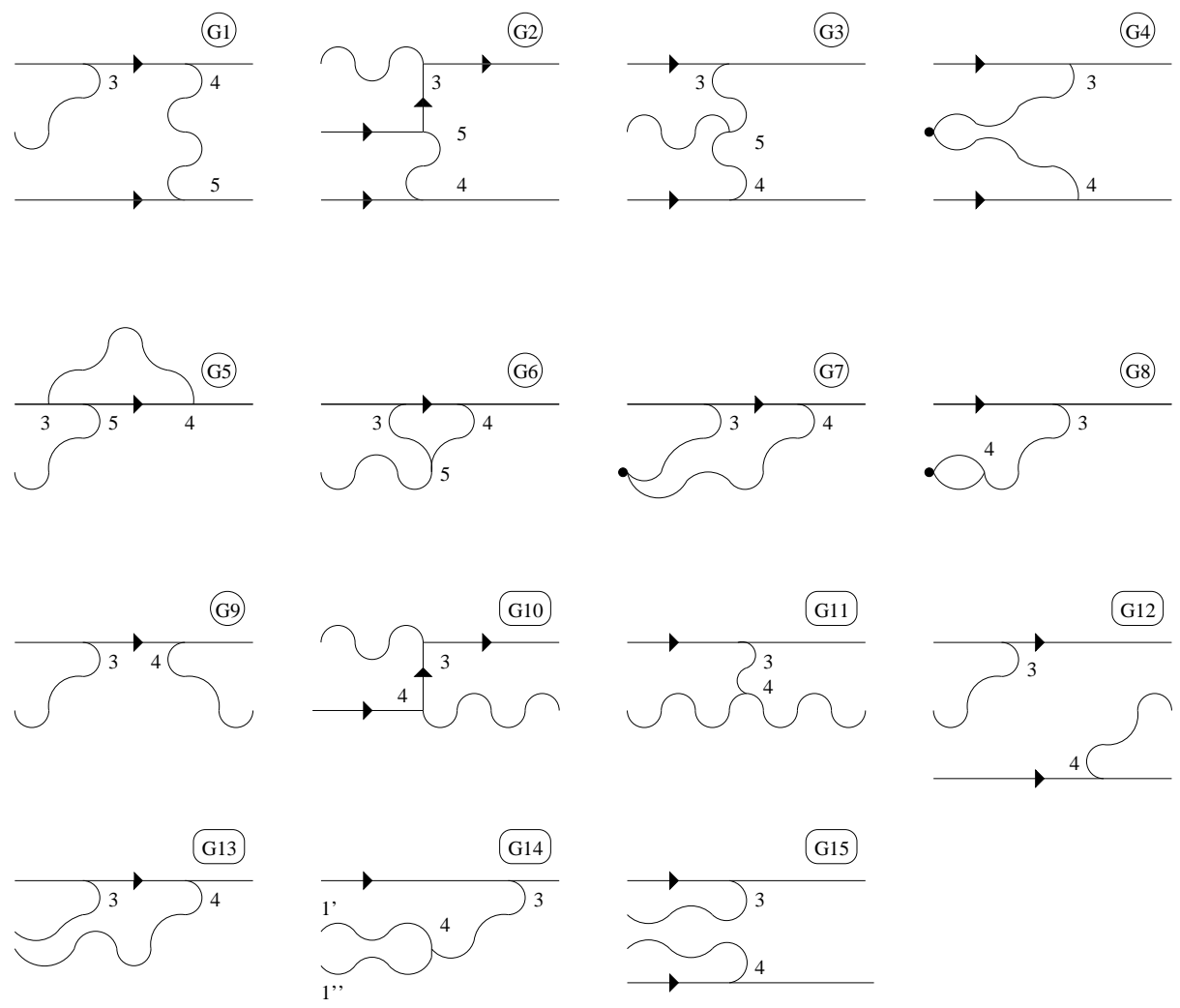

Figure 3. $O\left(g^{4}\right)$ graphs with connection lines.

Evaluation of the Grassmann- and $S U(2)$-integrations reduces the supergraphs to the following differential operators under the appropriate integrals:

$$
\begin{aligned}
G_{1}=(12)^{2} i[ & \left.\partial_{\mu 1} \square_{34} / 2-\partial_{\mu 34} \square_{1}\right] \\
G_{2}=(12)^{2} i[ & \left.\partial_{\mu 1} \square_{35} / 2+\partial_{\mu 23}\left(\partial_{14}+\partial_{15}\right)^{2}\right] \\
G_{3}=(12)^{2} i[ & \partial_{\mu 1} \square_{35} / 2+\partial_{\mu 23}\left(\partial_{14}+\partial_{15}\right)^{2}-\partial_{\mu 23} \square_{14} / 2 \\
& -\partial_{\mu 1} \square_{45} / 2-\partial_{\mu 24}\left(\partial_{13}+\partial_{15}\right)^{2}+\partial_{\mu 24} \square_{13} / 2 \\
& -\left(\partial_{\mu 23}-\partial_{\mu 24}\right) \square_{15} / 2 \\
& -\left(\partial_{\mu 23}-\partial_{\mu 24}\right) \square_{1} / 2 \\
& -\partial_{\mu 13}\left(\square_{1}+\square_{23}-2 \square_{24}\right) / 2+\partial_{\mu 14}\left(\square_{1}-2 \square_{23}+\square_{24}\right) / 2 \\
& \left.+\partial_{\mu 1}\left(\square_{23}-\square_{24}\right)-(12)\left(1^{-} 2^{-}\right) \partial_{\mu 15}\left(\square_{23}-\square_{24}\right)\right]
\end{aligned}
$$




$$
\begin{aligned}
& G_{4}=(12)^{2} i\left[-\left(\partial_{\mu 23}-\partial_{\mu 24}\right) / 2\right] \\
& G_{5}=(12) i\left[-\partial_{\mu 2} \square_{15}-(12)\left(1^{-} 2^{-}\right) \partial_{\mu 15} \square_{1}\right] \\
& G_{6}=(12) i\left[-\partial_{\mu 2} \square_{15}+\partial_{\mu 2} \square_{13} / 2-\partial_{\mu 1} \square_{1}+(12)\left(1^{-} 2^{-}\right) \partial_{\mu 15} \square_{1}\right] \\
& G_{7}=(12) i\left[\begin{array}{ll}
\partial_{\mu 2}
\end{array}\right] \\
& G_{8}=(12) i\left[\begin{array}{ll}
\partial_{\mu 2}
\end{array}\right] \\
& G_{9}=(12) \quad\left[-\partial_{\mu 34} \partial_{\nu 34}+\eta_{\mu \nu} \square_{34} / 4\right] \\
& G_{10}=(12)\left[\partial_{\nu 14} \partial_{\mu 23}+\partial_{\mu 34} \partial_{\nu 34}-\eta_{\mu \nu} \square_{34} / 4\right. \\
& \left.-\left(1+(12)\left(1^{-} 2^{-}\right)\right) \partial_{\mu 13} \partial_{\nu 24}\right] \\
& G_{11}=(12)\left[-\partial_{\nu 13} \partial_{\mu 23}+\partial_{\mu 1} \partial_{\nu 2}-\left(\partial_{\mu 14} \partial_{\nu 23}+\partial_{\mu 13} \partial_{\nu 24}\right) / 2\right. \\
& \left.+\eta_{\mu \nu}\left(\partial_{13}-\partial_{23}\right) \cdot\left(\partial_{14}-\partial_{24}\right) / 4-(12)\left(1^{-} 2^{-}\right) \partial_{\mu 14} \partial_{\nu 24}\right] \\
& G_{12}=(12)^{2}\left[\partial_{\mu 23} \partial_{\nu 14}\right] \\
& G_{13}=(12) \quad\left[\partial_{\mu 34} \partial_{\nu 2}+\eta_{\mu \nu} \square_{34} / 4\right] \\
& G_{14}=(12)\left[\partial_{\mu 23} \partial_{\nu 14}^{\prime}-\partial_{\nu 23} \partial_{\mu 14}^{\prime \prime}-\left(\partial_{\nu 23} \partial_{\mu 14}^{\prime}-\partial_{\mu 23} \partial_{\nu 14}^{\prime \prime}\right) / 2\right. \\
& \left.+\eta_{\mu \nu}\left(\partial_{13}-\partial_{23}\right) \cdot\left(\partial_{14}^{\prime}-\partial_{14}^{\prime \prime}\right) / 4\right] \\
& G_{15}=(12)^{2}\left[-\partial_{\mu 23} \partial_{\nu 24}\right]
\end{aligned}
$$

The combinatorics for $G_{4}$ is like for $G_{3}$ because the quadratic term in the connection is a commutator. The graphs come in a fixed sum in which $G_{4}$ compensates the $\square_{15}$ term in the expression for $G_{3}$. The box operators $\square_{23}, \square_{24}$ in (48) reduce the underlying $B U$ topology to the usual finite $f(1,2 ; 1,2)$. In all these terms the remaining derivative acts from the left and thus there are no poles. The $B U$ integral itself is in fact finite, too, as long as no double lines are introduced. We have checked that the $\square_{1}$ terms in the fifth line of (48) may be dropped as well. The integral is slightly more robust than $f$ in that the term $\left(\partial_{\mu 23}-\partial_{\mu 24}\right) \square_{1}$ is not singular either, although the derivatives act from the right. In conclusion,

$$
\begin{aligned}
G_{3}+G_{4}=(12)^{2} i[ & \partial_{\mu 1} \square_{35} / 2+\partial_{\mu 23}\left(\partial_{14}+\partial_{15}\right)^{2}-\partial_{\mu 23} \square_{14} / 2 \\
- & \left.\partial_{\mu 1} \square_{45} / 2-\partial_{\mu 24}\left(\partial_{13}+\partial_{15}\right)^{2}+\partial_{\mu 24} \square_{13} / 2+\ldots\right]
\end{aligned}
$$

where the dots indicate the omitted finite terms. Similarly, $G_{5}, G_{6}, G_{7}$ come in a fixed sum. On discarding finite terms:

$$
G_{5}+G_{6}+G_{7}=(12) i\left[-\partial_{\mu 2} \square_{15}+\partial_{\mu 2} \square_{13} / 2+\ldots\right]
$$

(The numbering of the points refers to $G_{5}, G_{6}$.) Owing to the box operators both terms can be fitted into the $O 2$ topology after joining a free line. It is interesting to note that the $\square_{13}, \square_{14}$ terms in (61) are of the same type, while the remaining terms are as in $G_{2}$. The graphs $G_{1} \ldots G_{7}$ seemingly always sum to zero if one end of the correlator is a BPS state. Diagram $G_{8}$ has the same combinatorics as the other graphs in the second line, but it is a separate piece in this sense.

In diagrams $G_{10}, G_{11}$ the potentially divergent pieces without $\eta$ are

$$
\begin{aligned}
G_{10} & =(12)\left[\partial_{\nu 14} \partial_{\mu 23}+\partial_{\mu 34} \partial_{\nu 34}+\ldots\right] \\
G_{11} & =(12)\left[-\partial_{\nu 13} \partial_{\mu 23}+\ldots\right]
\end{aligned}
$$


In our calculations the complete sum of graphs turned out to be finite within each class.

One last simplification concerns graph $G_{14}$ : for our purposes we may once again drop the $\eta$ term. Further, when the two indices are symmetrised

$$
G_{14}=(12)\left[\partial_{(\mu 23}\left(\partial_{\nu) 14}^{\prime}-\partial_{\nu) 14}^{\prime \prime}\right) / 2+\ldots\right] .
$$

We remark that diagram $G_{14}$ starts to contribute only at spin 4 .

All the integrals can be evaluated by the Mincer system just as the order $g^{2}$ examples: where there is only one matter line we join a second, free one. In this way $G_{1}, G_{5} \ldots G_{9}$, $G_{12} \ldots G_{14}$ are of topology $O 2$ (or derived of $O 2$ ) while the remaining diagrams belong to the $B U$ category. The rest of the algorithm is as before, although formula (41) must be adapted to the dimension of the three-loop integrals:

$$
(i q)^{\left(s_{l}+s_{r}\right)}\left(q^{2}\right)^{-3 \epsilon} \rightarrow \frac{3 \epsilon(1-4 \epsilon+\ldots)}{\pi^{2}}\left(-\partial_{1}\right)^{\left(s_{l}+s_{r}\right)} \frac{1}{\left(x_{12}^{2}\right)^{(2-4 \epsilon)}}
$$

\section{Conclusions and outlook}

We have discussed the descendant structure and renormalisation of the $\operatorname{sl}(2)$ sector operators of length three up to spin three. These are a $1 / 2$ BPS state $O$, a spin two operator $K_{6}$ belonging to the second lowest two-impurity BMN multiplet and two spin three primaries $V_{1}, V_{2}$ with identical scaling dimensions up to order $g^{4}$. The anomalous dimensions are

$$
K_{6}: \gamma_{1}=4 M, \gamma_{2}=-6 M^{2}, \quad V_{1}, V_{2}: \gamma_{1}=\frac{15}{2} M, \gamma_{2}=-\frac{225}{16} M^{2}
$$

with $M=N /\left(4 \pi^{2}\right)$. The result for $V_{1}, V_{2}$ is in exact agreement with [16], c.f. Table 2 .

The most difficult step in developing any higher-loop dilatation operator is to make it work when the spin-chain length is smaller than the maximum number of adjacent sites on which the operator can act ("wrapping"). From this perspective, the hardest test on any universal formula is to go from the shortest spin-chain length to operators with subsequently more sites in the trace. In the $s l(2)$ sector this would be the step from twist two to twist three, which we have checked for the Bethe-ansatz [16, initially at the lowest non-trivial spin.

The obvious continuation of our work is to investigate other mixing examples at higher spin as well as for operators with more elementary fields. The ultimate aim of the line of research is to set up a two-loop dilatation operator for the $s l(2)$ sector. It would be interesting to see whether the dilatation operator can be directly constructed from the elements of our renormalisation procedure by going back to the non-orthogonal basis $\left\{s_{1}, s_{2}, s_{3}\right\}$ defined in equation (11).

The renormalisation of $V_{1}$ is non-multiplicative: we have put $V_{1 \text { ren }}=\mathcal{Z}_{V} V_{1}-\mathcal{B} D_{z} K_{6}$ with a singular factor $\mathcal{B}$ that cannot be split into $b\left(g^{2}\right) \mathcal{Z}_{V}$, where $b\left(g^{2}\right)$ is some finite function. This example of orthogonalisation in dimensional regularisation closely parallels the discussion of the Konishi anomaly in [25].

Last, the limiting step in our current software is the manipulation of harmonic tensors, because for higher spin these involve very many terms. It would clearly be desirable to transfer a larger part of the calculation from Mathematica to Form. 


\section{Acknowledgements}

The author benefited from many useful discussions with G. Arutyunov, N. Beisert, V. Dippel, E. Sokatchev and M. Staudacher. He would also like to thank C. Jarczak for start up hints on xfig and G. Arutyunov, E. Sokatchev and M. Staudacher for comments on the manuscript.

\section{References}

[1] D. Berenstein, J. M. Maldacena and H. Nastase, "Strings in flat space and pp waves from N = 4 super Yang Mills," JHEP 0204 (2002) 013 arXiv:hep-th/0202021.

[2] J. A. Minahan and K. Zarembo, "The Bethe-ansatz for N = 4 super Yang-Mills," JHEP 0303 (2003) 013 arXiv:hep-th/0212208.

[3] N. Beisert, C. Kristjansen, J. Plefka and M. Staudacher, "BMN gauge theory as a quantum mechanical system," Phys. Lett. B 558, 229 (2003) arXiv:hep-th/0212269; N. Beisert, C. Kristjansen and M. Staudacher, "The dilatation operator of N = 4 super Yang-Mills theory," Nucl. Phys. B 664 (2003) 131 arXiv:hep-th/0303060.

[4] N. Beisert, J. A. Minahan, M. Staudacher and K. Zarembo, "Stringing spins and spinning strings," JHEP 0309 (2003) 010 arXiv:hep-th/0306139; N. Beisert, S. Frolov, M. Staudacher and A. A. Tseytlin, "Precision spectroscopy of AdS/CFT," JHEP 0310 (2003) 037 arXiv:hep-th/0308117.

[5] S. Frolov and A. A. Tseytlin, "Semiclassical quantization of rotating superstring in AdS(5) x S(5)," JHEP 0206, 007 (2002) arXiv:hep-th/0204226; "Multi-spin string solutions in AdS(5) x S**5," Nucl. Phys. B 668, 77 (2003) arXiv:hep-th/0304255; "Quantizing three-spin string solution in AdS(5) x S**5," JHEP 0307, 016 (2003) arXiv:hep-th/0306130; "Rotating string solutions: AdS/CFT duality in nonsupersymmetric sectors," Phys. Lett. B 570, 96 (2003) arXiv:hep-th/0306143; G. Arutyunov, S. Frolov, J. Russo and A. A. Tseytlin, "Spinning strings in AdS(5) x S**5 and integrable systems," Nucl. Phys. B 671, 3 (2003) arXiv:hep-th/0307191.

[6] N. Beisert, "The complete one-loop dilatation operator of $\mathrm{N}=4$ super Yang-Mills theory," Nucl. Phys. B 676, 3 (2004) arXiv:hep-th/0307015; N. Beisert and M. Staudacher, "The N = 4 SYM integrable super spin chain," Nucl. Phys. B 670, 439 (2003) arXiv:hep-th/0307042.

[7] N. Beisert, "The su(2|3) dynamic spin chain," Nucl. Phys. B 682 (2004) 487 arXiv:hep-th/0310252 ; B. Eden, C. Jarczak and E. Sokatchev, "A three-loop test of the dilatation operator in $\mathrm{N}=4$ SYM," arXiv:hep-th/0409009.

[8] N. Beisert, "Higher loops, integrability and the near BMN limit," JHEP 0309 (2003) 062 arXiv:hep-th/0308074.

[9] N. Beisert, V. Dippel and M. Staudacher, "A novel long range spin chain and planar N = 4 super Yang-Mills," JHEP 0407 (2004) 075 arXiv:hep-th/0405001. 
[10] D. Serban and M. Staudacher, "Planar N = 4 gauge theory and the Inozemtsev long range spin chain," JHEP 0406, 001 (2004) arXiv:hep-th/0401057.

[11] C. G. . Callan, H. K. Lee, T. McLoughlin, J. H. Schwarz, I. Swanson and X. Wu, "Quantizing string theory in $\operatorname{AdS}(5) \times \mathrm{S}^{* *} 5$ : Beyond the pp-wave," Nucl. Phys. B 673, 3 (2003) arXiv:hep-th/0307032; C. G. . Callan, T. McLoughlin and I. Swanson, "Holography beyond the Penrose limit," Nucl. Phys. B 694, 115 (2004) arXiv:hep-th/0404007; "Higher impurity AdS/CFT correspondence in the near-BMN limit," Nucl. Phys. B 700, 271 (2004) arXiv:hep-th/0405153; T. McLoughlin and I. Swanson, "N-impurity superstring spectra near the pp-wave limit," Nucl. Phys. B 702, 86 (2004) arXiv:hep-th/0407240.

[12] G. Arutyunov, S. Frolov and M. Staudacher, "Bethe ansatz for quantum strings," JHEP 0410, 016 (2004) arXiv:hep-th/0406256.

[13] G. Arutyunov and M. Staudacher, "Matching higher conserved charges for strings and spins," JHEP 0403 (2004) 004 arXiv:hep-th/0310182; "Two-loop commuting charges and the string / gauge duality," arXiv:hep-th/0403077.

[14] V. A. Kazakov, A. Marshakov, J. A. Minahan and K. Zarembo, "Classical / quantum integrability in AdS/CFT," JHEP 0405, 024 (2004) arXiv:hep-th/0402207.

[15] V. A. Kazakov and K. Zarembo, "Classical / quantum integrability in non-compact sector of AdS/CFT," JHEP 0410, 060 (2004) arXiv:hep-th/0410105.

[16] M. Staudacher, "The factorized S-matrix of CFT/AdS," arXiv:hep-th/0412188.

[17] A. V. Kotikov, L. N. Lipatov, A. I. Onishchenko and V. N. Velizhanin, "Three-loop universal anomalous dimension of the Wilson operators in N $=4$ SUSY Yang-Mills model," Phys. Lett. B 595 (2004) 521 arXiv:hep-th/0404092.

[18] S. Moch, J. A. M. Vermaseren and A. Vogt, "The three-loop splitting functions in QCD: The non-singlet case," Nucl. Phys. B 688 (2004) 101 arXiv:hep-ph/0403192.

[19] A. Galperin, E. Ivanov, S. Kalitsyn, V. Ogievetsky and E. Sokatchev, "Unconstrained $\mathrm{N}=2$ matter, Yang-Mills and supergravity theories in harmonic superspace," Class. Quant. Grav. 1 (1984) 469; A. S. Galperin, E. A. Ivanov, V. I. Ogievetsky and E. S. Sokatchev, "Harmonic superspace," Cambridge, UK: Univ. Pr. (2001) 306 p.

[20] W. Siegel, "Supersymmetric dimensional regularization via dimensional reduction," Phys. Lett. B 84 (1979) 193; M. T. Grisaru, W. Siegel and M. Rocek, "Improved methods for supergraphs," Nucl. Phys. B 159 (1979) 429.

[21] K. G. Chetyrkin, A. L. Kataev and F. V. Tkachov, "New approach to evaluation of multiloop feynman integrals: the Gegenbauer polynomial x space technique," Nucl. Phys. B 174 (1980) 345; K. G. Chetyrkin and F. V. Tkachov, "Integration by parts: the algorithm to calculate beta functions in 4 loops," Nucl. Phys. B 192 (1981) 159; D. I. Kazakov, "The method of uniqueness, a new powerful technique for multiloop calculations," Phys. Lett. B 133 (1983) 406; S. A. Larin, F. V. Tkachov and J. A. M. Vermaseren, "The FORM version of MINCER," NIKHEF-H-91-18. 
[22] I. Y. Park, A. Tirziu and A. A. Tseytlin, "Spinning strings in $\mathrm{AdS}_{5} \times \mathrm{S}^{5}$ : one-loop correction to energy in SL(2) sector," arXiv:hep-th/0501203.

[23] E. S. Fradkin and M. Y. Palchik, "Conformal quantum field theory in D-dimensions," Dordrecht, Netherlands: Kluwer (1996) 461 p. (Mathematics and its applications. 376)

[24] S. Penati, A. Santambrogio and D. Zanon, "Two-point functions of chiral operators in N = 4 SYM at order g**4," JHEP 9912 (1999) 006 arXiv:hep-th/9910197; "More on correlators and contact terms in N =4 SYM at order g**4," Nucl. Phys. B 593 (2001) 651 arXiv:hep-th/0005223.

[25] B. Eden, C. Jarczak, E. Sokatchev and Y. S. Stanev, "Operator mixing in N = 4 SYM: The Konishi anomaly revisited," arXiv:hep-th/0501077.

[26] P. S. Howe, E. Sokatchev and P. C. West, "3-point functions in N = 4 Yang-Mills," Phys. Lett. B 444 (1998) 341 arXiv:hep-th/9808162; B. Eden, P. S. Howe, C. Schubert, E. Sokatchev and P. C. West, "Four-point functions in $\mathrm{N}=4$ supersymmetric Yang-Mills theory at two loops," Nucl. Phys. B 557 (1999) 355 arXiv:hep-th/9811172; B. Eden, P. S. Howe, C. Schubert, E. Sokatchev and P. C. West, "Simplifications of four-point functions in $\mathrm{N}=4$ supersymmetric Yang-Mills theory at two loops," Phys. Lett. B 466 (1999) 20 arXiv:hep-th/9906051; P. S. Howe, C. Schubert, E. Sokatchev and P. C. West, "Explicit construction of nilpotent covariants in N $=4$ SYM," Nucl. Phys. B 571 (2000) 71 arXiv:hep-th/9910011. 